\title{
ANÁLISE DO CONTRASTE DA PENALIDADE E DA RECOMPENSA (PRC): identificando atributos de qualidade em serviços de academias
}

\section{1- Jucelia Appio Tibola*}

Doutoranda em Administração pela Universidade Positivo (UP).

Professora da Horus Faculdades.

juceliaappio@yahoo.com.br

http://lattes.cnpq.br/1451426210695510

\section{2- Aletéia Silva}

Mestre em Administração pela Universidade Regional de Blumenau (FURB)

Professora da Faculdade de Ciênicas Sociais Aplicadas de Cascavel (UNIVEL)

aleteia@univel.br

http://lattes.cnpq.br/8146150984908773

\section{3- Valter Afonso Vieira}

Doutorado em Administração de Empresas pela Universidade de Brasília (UNB) Professor do curso de Administração da Universidade Federal do Paraná (UFPR)

valterafonsovieira@yahoo.com

http://lattes.cnpq.br/4208847621549079

\section{4- Gérson Tontini}

Doutorado em Engenharia Mecânica pela Universidade Federal de Santa Catarina (UFSC)

Professor dos programas de Mestrado e Doutorado em Administração da Universidade Regional de Blumenau (FURB)

tontini@furb.br

http://lattes.cnpq.br/1679721959381613 


\title{
ANÁLISE DO CONTRASTE DA PENALIDADE E DA RECOMPENSA (PRC): IDENTIFICANDO ATRIBUTOS DE QUALIDADE EM SERVIÇOS DE ACADEMIAS
}

\section{RESUMO}

A grande concorrência enfrentada pelas empresas prestadoras de serviço faz com que exista uma exigência de qualidade em níveis cada vez mais altos. Para isso, os esforços e recursos precisam ser direcionados da forma mais objetiva possível. O método utilizado nesta pesquisa, a Análise do Contraste da Penalidade e da Recompensa (PRC), permite que os empresários conheçam quais atributos do serviço prestado devem ser melhorados e quais devem ser mantidos. Duas academias foram identificadas como objeto de estudos. Os resultados mostram que os clientes consideram a presteza e as instalações físicas das academias como obrigatórios para obtenção de satisfação geral.

\section{Palavras-Chave}

Serviços. Atributos. Qualidade. Análise do Contraste da Penalidade e da recompensa (PRC).

\section{AN ANALYSIS OF THE PENALTY AND REWARD CONTRASTS: IDENTIFYING THE SERVICE QUALITY ATTRIBUTES ON ACADEMIES}

\begin{abstract}
The competition faced by large companies demands high quality services and perfection levels. To achieve this superior performance, efforts and resources are addressed as goals. The method used in this research, the Analysis of Contrast Penalty and Reward (ACPR), allows businesses to know which services attributes should be improved (revised) and which should be maintained. Two academies were analyzed as sample size. The results showed that customers consider the academies' readiness and physical facilities as required to obtain higher levels of satisfaction.
\end{abstract}

\section{Keywords}

Services. Attributes. Quality. Contrast Analysis of Penalty and Reward (PRC). 


\section{Introdução}

A Revolução Industrial ocorrida no século XX moldou a sociedade pós-moderna que grande parte do mundo vivencia atualmente. A principal característica desta sociedade é o capitalismo descomedido. Durante o desenvolvimento desta estrutura aconteceu um aumento significativo de empresas prestadoras de serviços dos mais variados possíveis.

Assim que as precursoras começaram a se consolidar, mais empresas foram surgindo, criando grande concorrência no mercado. Hoje, a maior preocupação de grande parte delas - as que sobrevivem - vai além da oferta de serviços satisfatórios. De fato, as empresas estão tendo que aumentar os níveis de qualidade em todos os aspectos para serem competitivas. Sobre este contexto, Berry (1996) salienta que a qualidade deve ser mantida em níveis bastante altos e que, para isso, não é necessário gastar grandes quantias em dinheiro, mas realizar planejamento e atividades diárias para oferecer constante melhoria. Mas como identificar o que deve ser mantido e o que deve ser melhorado?

Para responder este questionamento, modelos de avaliação de atributos de qualidade têm sido estudados no sentido de amparar e de dar conotação cientifica à mensuração, evoluindo de tempos em tempos para modelos cada vez mais precisos. Entre os principais estudos estão aqueles realizados por autores internacionais, como Grönroos (1984), Kano (1984), Parasuraman, Zeithaml \& Berry (1985; 1988), Brandt (1987), Bolton e Drew (1991), Anderson e Mital (2000); Ting e Chen (2002), Matzler, Bailon, Hinterhuber, Renzl \&Pichler (2004), e nacionais, como Tontini (1996; 2003a; 2003b; 2003c), Tontini e Silveira (2005), e Picolo e Tontini (2006).

Percebe-se, no entanto, que estudos dos atributos de qualidade voltados para academias de ginástica não se revelam na literatura. A maior parte das pesquisas sobre satisfação de clientes e qualidade em serviços, em bases de dados nacionais e internacionais, está direcionada às instituições de ensino. Tal fato ocorre, provavelmente, pela facilidade de acesso aos respondentes na aplicação dos questionários.

Com este argumento, o presente artigo direciona-se para a mensuração da qualidade em academias de ginástica e pretende verificar a satisfação dos clientes com as cinco dimensões da qualidade apontadas pela escala SERVQUAL (aspectos tangíveis, confiabilidade, presteza, segurança e empatia) e a relação entre o desempenho dos atributos com a satisfação geral dos clientes de duas academias de ginástica de uma cidade do Sudoeste do Paraná.

Os dados serão tratados e analisados por meio da análise do contraste da penalidade e da recompensa (PRC) e pela Matriz de Importância e Desempenho. Desta forma, será possível identificar entre as cinco dimensões quais são as consideradas mais importantes pelos clientes e quais estão obtendo desempenho satisfatório, ou precisam receber especial atenção para realização de melhorias. Estes modelos permitem evitar o desperdício de investimentos em atributos que não aumentarão a satisfação, e focá-los naqueles que são mais bem percebidos pelos clientes.

O artigo está estruturado da seguinte forma: a primeira parte traz uma revisão de literatura sobre escala SERVQUAL, dimensões da qualidade, matriz de importância vs, desempenho, de modo a ressaltar origens e definições. Na segunda parte a metodologia de pesquisa é apresentada. Na etapa seguinte, os dados são analisados e discutidos, primeiramente, a partir das dimensões da escala SERVQUAL, em seguida, com a análise do PRC e, por fim, por meio da matriz de importância vs. desempenho das dimensões de pesquisa. As considerações finais concluem o artigo.

\section{Escala SERVQUAL e as dimensões da qualidade}

Parasuraman et al. (1985) realizaram uma pesquisa qualitativa exploratória com executivos de quatro empresas americanas prestadoras de serviços em diferentes áreas, como banco varejista, cartão de crédito, corretagem de ações, reparo e manutenção de produtos. Os pesquisadores procuraram levantar dados que indicassem:

- os principais atributos de empresas prestadoras de serviços,

- os principais problemas,

- a forma com que os consumidores percebem os diferentes atributos da qualidade em serviços,

- a existência de discrepância entre a percepção do consumidor e a dos responsáveis pelo marketing das empresas, e 
- a possibilidade de geração de um modelo que explique a qualidade do serviço a partir da conexão entre a percepção do consumidor e a dos responsáveis pelo marketing.

Além das entrevistas com os executivos, Parasuraman et al. (1985) entrevistaram doze grupos de foco para um conhecimento mais aprofundado sobre as questões acima mencionadas. Após analisar o conteúdo das entrevistas com os executivos e com os grupos de foco, os pesquisadores conseguiram chegar a 97 itens, divididos em dez categorias de atributos, denominadas de dimensões da qualidade. As dimensões são: confiabilidade, presteza, competência, acessibilidade, cortesia, comunicação, credibilidade, segurança, compreensão/conhecimento do cliente e aspectos tangíveis (evidências físicas relacionadas ao serviço).

Em 1988, Parasuraman et al. realizaram outra pesquisa, desta vez com 200 pessoas que utilizavam determinados serviços freqüentemente ou que haviam utilizado há pouco tempo. As informações coletadas foram processadas e, baseados nos coeficientes de correlação dos itens e na importância subjetivamente analisada, ficaram 22 itens, divididos em 05 dimensões da qualidade. Este instrumento é denominado de escala SERVQUAL e tem por objetivo verificar como os consumidores avaliam a qualidade dos serviços comparada à sua própria expectativa.

As cinco dimensões que representam a escala SERVQUAL estão listadas e caracterizadas a seguir (Parasuraman et al., 1988, Bateson e Hoffman, 2001; Fitzsimmons e Fitzsimmons, 2001):

- Aspectos tangíveis: referem-se à aparência das instalações físicas, dos equipamentos, do pessoal e dos materiais para comunicação. A condição do ambiente é uma evidência tangível do cuidado e da atenção aos detalhes exibidos pelo fornecedor do serviço. A dimensão de itens tangíveis do SERVQUAL compara as expectativas dos consumidores com o desempenho da empresa no que diz respeito à capacidade da empresa de gerenciar seus itens tangíveis.

- Confiabilidade: é a capacidade de prestar o serviço prometido com confiança e exatidão. O desempenho de um serviço confiável é uma expectativa do cliente e significa um serviço cumprido no prazo, sem modificações e sem erros. Em geral, a confiabilidade reflete a consistência e a certeza do desempenho da empresa. Em muitos casos, o consumidor está pronto para gastar dinheiro se o prestador de serviço desempenhar a transação que prometeu.

- Presteza/Responsabilidade/Receptividade: é a vontade ou disposição para auxiliar os clientes e fornecer o serviço prontamente (pronto atendimento). Se ocorrer uma falha em um serviço, a capacidade para recuperá-la rapidamente e com profissionalismo pode gerar muitas percepções positivas da qualidade. Como tal, esta dimensão do SERVQUAL diz respeito ao desejo e/ou disponibilidade dos funcionários para produzir um serviço.

- Segurança: está relacionada ao conhecimento e à cortesia dos funcionários, bem como sua capacidade de transmitir confiança e confidencialidade. Inclui competência para realizar o serviço, cortesia e respeito ao cliente, comunicação efetiva com o cliente e a idéia de que o funcionário está realmente interessado em fazer o melhor para o cliente.

- Empatia: é demonstrar interesse e atenção personalizada aos clientes. Inclui algumas características, tais como, acessibilidade, sensibilidade e esforço para entender as necessidades dos clientes.

\section{Matriz de importância vs desempenho}

Originalmente introduzida por Martilha e James (1977), a matriz de importância vs desempenho é uma técnica de análise muito usada para identificação de qualidade e desempenho dos atributos percebidos pelos clientes. Por meio da interação entre essas duas dimensões, é possível formular estratégias de gerenciamento da satisfação dos clientes para melhor alocação dos recursos. (Matzler et al., 2004).

Tipicamente, os dados provenientes de pesquisas de satisfação são utilizados para construir uma matriz bidimensional, na qual a importância é demonstrada no eixo $y$ e desempenho no eixo $x$. Os atributos provenientes da pesquisa são agrupados em uma matriz com quatro quadrantes. $O$ atributo que se situar no Quadrante I terá alta importância e alto desempenho, sustentando uma possível vantagem competitiva, devendo a empresa manter seu trabalho. Um atributo que tiver alta importância, mas baixo desempenho exige atenção imediata (Quadrante II). Para aumentar a satisfação geral, a empresa deve concentrar-se nestes atributos, pois ignorá-los pode causar uma ameaça. O Quadrante III contém atributos com baixa importância e baixo desempenho, não é necessário concentrar esforços adicionais nestes atributos por 
serem de baixa prioridade. No Quadrante IV apresentam-se os atributos com alto desempenho, mas baixa importância; isto significa que a empresa deve melhor aproveitar seus recursos em outros atributos.

A importância dos atributos pode ser dada pela importância declarada explicitamente pelos clientes, ou pela importância derivada estatisticamente (Garver, 2003). Na importância declarada, os consumidores tendem a dar alta importância para quase todos os atributos. Já na importância derivada estatisticamente, os consumidores tendem a diferenciar mais os atributos; porém o método estatístico indica relação linear entre satisfação com os atributos e a satisfação geral (Tontini e Silveira, 2005).

Neste estudo, para elaboração da matriz de importância vs desempenho, será utilizada a importância derivada estatisticamente.

\section{Metodologia de pesquisa}

A presente pesquisa é composta por critérios de amostra não probabilística, com 63 clientes pertencentes às duas maiores academias de ginástica localizadas na cidade de Francisco Beltrão-PR. Para resguardar suas identidades, foram denominadas de academia ALFA e academia BETA. Cabe salientar que a amostra de 63 clientes possui limitações por possuir erro amostral superior a 10\%, entretanto, como os clientes freqüentam as academias em média 3 a 4 vezes por semana, considerou-se esta amostra como adequada para realização do estudo. Permitiu-se uma margem de erro amostral de $12,70 \%$ para a academia ALFA e $14,80 \%$ para a academia BETA (Figura 1), com intervalo de confiança de $95 \%$ a $99 \%$, conforme orientação de Hair Jr. et al. (2009).

Figura 1: População e amostra de pesquisa

\begin{tabular}{|lr|}
\hline \multicolumn{2}{|c|}{ Cálculo da amostra - Academia ALFA } \\
\hline Erro amostral tolerável & $12,70 \%$ \\
População & 82 \\
Amostra & 35 \\
\hline
\end{tabular}

\begin{tabular}{|lr|}
\hline \multicolumn{2}{|c|}{ Cálculo da amostra - Academia BETA } \\
\hline Erro amostral tolerável & $14,80 \%$ \\
População & 73 \\
Amostra & 28 \\
\hline
\end{tabular}

Fonte: dados da pesquisa, 2008

Para o desenvolvimento desta pesquisa, utilizou-se um questionário estruturado, adaptado de Parasuraman et al. (1988). Os atributos pesquisados foram sintetizados e agrupados com base nas determinantes de qualidade: aspectos tangíveis, confiabilidade, presteza, segurança e empatia.

O questionário foi dividido em duas partes. A primeira, composta de 15 questões usando uma escala variando de Discordo Totalmente (1) até Concordo Totalmente (7), identificou a opinião dos clientes em relação aos serviços de academias em geral, sendo eles: equipamentos modernos; instalações físicas; aparência física do colaborador (vestuário e limpeza); solidariedade e segurança transmitida pelos colaboradores; programas que atinjam os objetivos esperados; prestação de serviços conforme prometido; disposição imediata para ajudar; ajuda aos clientes; prontidão no atendimento; colaboradores confiáveis; educação dos colaboradores; colaboradores recebem suporte da academia; atenção individual aos clientes; identificação das necessidades do cliente; e horário conveniente para todos. Na segunda etapa, as perguntas da primeira parte foram reaplicadas, porém, focadas no desempenho atual dos serviços prestados pelas academias. Duas perguntas adicionais foram aplicadas com o intuito de identificar o nível de satisfação geral dos clientes das duas academias.

Um pré-teste com 11 clientes da academia ALFA e 4 da academia BETA foi realizado para ajustes no questionário. Após adequação do instrumento de coleta, realizou-se a pesquisa in loco, entre os dias 02 e 17 de setembro de 2008. Para a coleta de dados, solicitou-se que os funcionários distribuíssem os questionários aos clientes e, após preenchimento, os mesmos deveriam colocar em uma urna lacrada, para resguardar o anonimato dos respondentes.

Durante a tabulação dos resultados, sete questionários da academia ALFA e três da academia BETA foram eliminados por possuírem respostas inválidas e inconsistentes.

Na próxima seção os resultados da pesquisa serão apresentados e, junto às análises, serão expostas tabelas para uma melhor visualização. A análise quantitativa contou com o suporte do software SPSS. 


\section{Resultados obtidos}

Para melhor compreensão dos resultados obtidos, este tópico será dividido em quatro partes. Na primeira, apresenta-se a caracterização da amostra pesquisada; na segunda parte, são apresentados os resultados obtidos com aplicação da escala SERVQUAL; a terceira parte consiste na análise do Contraste da Penalidade e da Recompensa (PRC); e, ao final, a matriz de importância vs desempenho.

\subsection{Caracterização da amostra}

Quanto ao gênero dos respondentes, $62,90 \%$ são mulheres e $37,10 \%$ são homens, caracterizando uma amostra com predominância feminina. Em termos de idade, a maior concentração dos respondentes tem entre 21 e 35 anos (46,77\%), seguido dos respondentes entre 36 e 50 anos (25,81\%). Em termos de estado civil, houve maior concentração de solteiros, que representaram $54,84 \%$ da amostra, seguidos por casados, com $40,32 \%$.

\subsection{Análise das dimensões da escala servqual}

Foram escolhidos os 15 principais atributos dos 22 que compunham o instrumento SERVQUAL. Estes atributos foram enquadrados em 5 dimensões da qualidade, conforme disposto na Figura 2 (Parasuraman et al.,1988).

Figura 2: Dimensões da escala SERVQUAL

\begin{tabular}{|c|c|}
\hline \multicolumn{2}{|r|}{ Variáveis pesquisadas para as cinco dimensões qualidade } \\
\hline Aspectos Tangíveis & $\begin{array}{l}1 \text { - As academias devem ter equipamentos modernos. } \\
2 \text { - As instalações físicas devem ser visualmente atrativas. } \\
3 \text { - Os seus colaboradores devem estar bem vestidos e aparentando limpeza. }\end{array}$ \\
\hline Confiabilidade & $\begin{array}{l}4 \text { - As academias devem ser solidárias e deixar os clientes seguros quando estes têm } \\
\text { algum problema. } \\
5 \text { - As academias devem ter programas que atingem os resultados esperados. } \\
6 \text { - As academias devem fornecer o serviço conforme prometido. }\end{array}$ \\
\hline Presteza & $\begin{array}{l}7 \text { - Os colaboradores das academias têm que estar sempre disponíveis para ajudar os } \\
\text { clientes. } \\
8 \text { - Os colaboradores das academias devem ajudar os clientes. } \\
9 \text { - colaboradores das academias devem responder prontamente aos pedidos dos } \\
\text { clientes. }\end{array}$ \\
\hline Segurança & $\begin{array}{l}10 \text { - Os clientes devem poder acreditar nos colaboradores de academias. } \\
11 \text { - Os colaboradores de academias devem ser educados. } \\
12 \text { - Os colaboradores devem ter suporte adequado das academias onde trabalham } \\
\text { para cumprir suas tarefas corretamente. }\end{array}$ \\
\hline Empatia & $\begin{array}{l}13 \text { - As academias precisam dar atenção individual aos clientes. } \\
14 \text { - Os colaboradores das academias devem saber quais são as necessidades dos } \\
\text { clientes. } \\
15 \text { - O horário de funcionamento das academias tem que ser conveniente para todos os } \\
\text { clientes. }\end{array}$ \\
\hline
\end{tabular}

Fonte: adaptado de Parasuraman et al. (1988)

As respostas dos clientes a respeito do desempenho atual com os atributos foram processadas, para que fosse possível verificar a correlação entre cada uma delas e a satisfação geral com as academias. Nessa primeira análise, o grau de correlação foi baixo e algumas questões ficaram posicionadas em mais de uma dimensão.

Como forma de melhorar a representação dos dados, realizou-se a análise dos componentes principais (ACP) das variáveis. A princípio, as 15 questões estavam divididas em 5 dimensões, sendo: aspectos tangíveis, confiabilidade, presteza, segurança e empatia. Com a $\mathrm{ACP}$, as dimensões foram reduzidas para 3. Conforme a Figura 3, as questões Q5, Q14, 15, Q13, Q6, Q12 e Q9 ficaram agrupadas na dimensão denominada presteza. A segunda dimensão denominada foi segurança e agrupou as questões Q7, Q11, Q10, Q8, Q3, Q4. A terceira dimensão agrupou as questões Q2 e Q1 e está relacionada aos itens denominados de aspectos tangíveis das academias.

Quando observados os dados da pesquisa, percebe-se que as dimensões foram reagrupadas conforme a compatibilidade entre as características dos atributos: 
Dimensão Presteza: confiança dos clientes para com os programas da academia; conhecimento dos colaboradores em relação às necessidades dos clientes; horários convenientes para os clientes; atenção individualizada; fornecimento do serviço conforme prometido; suporte adequado da academia para seus colaboradores poderem cumprir suas tarefas e; resposta imediata dos colaboradores aos pedidos dos clientes.

Dimensão Segurança: atendimento imediato por parte dos colaboradores; colaboradores educados; credibilidade dos colaboradores; os colaboradores estão sempre dispostos a ajudar; os colaboradores são bem vestidos e aparentam limpeza e; quando os clientes têm algum problema com a academia ela é solidária e deixa-os seguros.

Dimensão Aspectos Tangíveis: agrupamentos de atributos tangíveis, tais como equipamentos modernos e instalações físicas visualmente atrativas.

Figura 3: Análise em Componentes Principais das dimensões de pesquisa.

\begin{tabular}{|l|r|r|r|}
\hline & \multicolumn{3}{|c|}{ Matriz de Componentes (a) } \\
\hline & 1 & 2 & 3 \\
\hline Q5 &, 867 & & \\
Q14 &, 805 & \\
Q15 &, 700 & \\
Q13 &, 689 & \\
Q6 &, 653 & & \\
Q12 &, 574 & & \\
Q9 &, 501 &, 735 & \\
Q7 & &, 728 & \\
Q11 & &, 694 & \\
Q10 & &, 653 & \\
Q8 & &, 646 & \\
Q3 & &, 624 & \\
Q4 & & & \\
Q2 & & & \\
Q1 & & & \\
\hline
\end{tabular}

* Método de Extração: Análise dos Componentes Principais. Método de Rotação: Varimax com normalização de Kayser. Fonte: dados da pesquisa, 2008

Este reagrupamento foi utilizado na seqüência do trabalho para a análise PRC - Contraste da Penalidade e Recompensa, com o objetivo de identificar as relações lineares e não lineares entre a satisfação com cada uma das dimensões e a satisfação geral.

Após novo agrupamento das dimensões, procedeu-se a análise de correlação dos dados. A Figura 4 apresenta os coeficientes de correlação de Pearson, $r$, entre as variáveis e o nível da significância. Observando os dados, percebe-se a existência de correlações fortes e significativas entre as variáveis, tendo o valor de $\mathrm{KMO}=0,832$. A recomendação de valor ideal de Maroco (2003) é de 0,70; portanto, o novo agrupamento das dimensões da pesquisa possui correlação satisfatória.

Um instrumento de coleta de dados (questionário) é considerado confiável se sua aplicação resulta em escores coerentes. Ou seja, a confiabilidade refere-se à coerência das descobertas da pesquisa. Os itens deverão ser correlacionados, pois quanto maior a correlação, maior a confiabilidade da escala. Este processo submete os dados ao teste de confiabilidade denominado alpha de Cronbach, que varia de 0 a 1 , sendo que os valores dentre $\alpha=0,60$ e $\alpha=0,70$ são considerados limítes de aceitabilidade (Hair JR. et al., 2005).

No caso da pesquisa com as academias, utilizando a análise do alpha de Cronbach, foi possível examinar a inter-relação entre as variáveis latentes. Quando avaliada a intensidade da associação das dimensões de pesquisa, os dados indicaram intensidade de associação muito boa para as dimensões presteza $(0,871)$ e segurança $(0,842)$, e associação moderada para a dimensão aspectos tangíveis $(0,641)$. (Ver Figura 5). 
Figura 4: Adequação da Amostra de KMO e Teste de esfericidade de Bartlett's

Fonte: dados da pesquisa, 2008

\begin{tabular}{|c|c|r|}
\hline \multicolumn{2}{|c|}{ Adequação da Amostra de Kaiser-Meyer-Olkin } & 0,832 \\
\hline $\begin{array}{c}\text { Teste de esfericidade } \\
\text { de Bartlett's }\end{array}$ & $\begin{array}{c}\text { Qui-quadrado } \\
\text { aproximado }\end{array}$ & 459,977 \\
\cline { 2 - 3 } & DF & 105 \\
\cline { 2 - 3 } & Sig. & 0,000 \\
\hline
\end{tabular}

Figura 5: Análise de confiabilidade Alfa de Cronbach

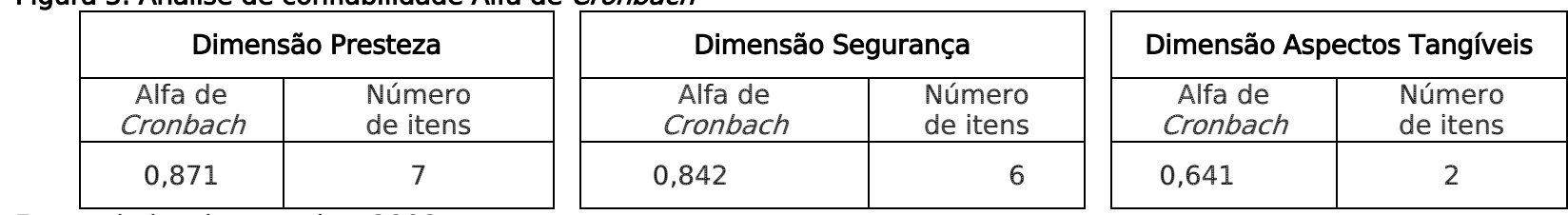

Fonte: dados da pesquisa, 2008

Para a análise de regressão, as dimensões presteza, segurança e aspectos tangíveis foram consideradas variáveis preditoras (variáveis independentes), já a satisfação geral foi considerada como variável dependente. A análise de regressão apresentou um $R^{2}=0,675$; sendo assim, os dados indicam que $67,5 \%$ da variabilidade total da satisfação geral pode ser explicada pelas variáveis independentes presteza, segurança e aspectos tangíveis.

Figura 6: Análise de regressão das dimensões de pesquisa

\begin{tabular}{|c|c|c|c|c|}
\hline Modelo $(b)$ & $R$ & $R^{2}$ & $R^{2}$ ajustado & Erro padrão da estimativa \\
\hline 1 & $0,83(a)$ & 0,691 & 0,675 & 0,5699 \\
\hline
\end{tabular}

* a) Previsores: (Constante), Aspectos Tangíveis, Presteza, Segurançao e b)Variável dependente: Satisfação. Fonte: dados da pesquisa, 2008

A análise dos valores absolutos dos coeficientes de regressão padronizados permitiu identificar que as variáveis presteza $(0,587)$ e segurança $(0,558)$ apresentaram as maiores contribuições relativas para explicar a variável dependente satisfação geral. Quando observados os valores da estatística de $t$, confirmou-se esta afirmação: presteza possui maior valor, sendo $t=8,048$ e segurança possui $t=7,650$. Isso significa que estas duas dimensões afetam em maior proporção a variável satisfação geral.

Figura 7: Análise de regressão das dimensões de pesquisa para o modelo PRC

\begin{tabular}{|c|c|c|c|c|}
\hline Modelo(b) & $R$ & $R^{2}$ & $R^{2}$ ajustado & Erro padrão da estimativa \\
\hline 1 &, $872(a)$ & 0,760 & 0,734 &, 51601173 \\
\hline
\end{tabular}

*' a) Preditores: (Constante), PrestezaMa, SegurMa, Asp. TangiveisMa, PrestezaMe, SegurMe, Asp. TangiveisMe.

$* 2$ b) Variável dependente: Satisfação

Fonte: dados da pesquisa, 2008

\subsection{Análise do PRC para o caso das academias}

Segundo Picolo e Tontini (2006), a análise do contraste da penalidade e da recompensa (PRC) identifica as relações lineares e não lineares entre a satisfação geral e a satisfação com os atributos. Com esta identificação, as empresas podem investir em melhorias que possam trazer maior grau de satisfação dos clientes. A análise acontece a partir dos coeficientes $\square$ em casos de multiatributos, com a equação de regressão: $Y=\square+\square \times 1$ pos $+\square \mathbb{D} 2$ neg $+\ldots+\square X$ Xnpos $+\square$ Xnneg, onde $Y$ é a satisfação geral e " $X_{n}$ " é a satisfação com cada atributo (Mittal, Ross \& Baldasare, 1998).

$\mathrm{Na}$ pesquisa realizada por Picolo e Tontini (2006), este procedimento foi utilizado para verificar a influência de cada um dos 16 atributos na satisfação geral dos clientes de dois supermercados. Os autores concluíram que a satisfação geral estava recebendo maior influência dos atributos relacionados a procedimentos de operação do que atributos relacionados à estrutura física. 
No caso das academias, o método foi realizado conforme atributos avaliados pelos clientes e agrupados em três dimensões (presteza, segurança e aspectos tangíveis). Os dados foram ajustados, sendo que os atributos com média baixa (menor que zero) receberiam penalidade e os atributos acima da média (maior que zero) receberiam recompensa. Por meio da aplicação do método PRC, foram identificados atributos relevantes para aumentar a satisfação geral dos clientes das academias pesquisadas.

Figura 8: Análise de regressão das dimensões de pesquisa para o modelo PRC

\begin{tabular}{|c|c|c|c|c|}
\hline Modelo(b) & $R$ & $R^{2}$ & $R^{2}$ ajustado & Erro padrão da estimativa \\
\hline 1 &, $872(a)$ & 0,760 & 0,734 &, 51601173 \\
\hline
\end{tabular}

$*^{* 1}$ a) Preditores: (Constante), Prestezama, Segurma, Asp. Tangiveisma, Prestezame, Segurme, Asp. Tangiveisme.

*2 b) Variável dependente: Satisfação

Fonte: dados da pesquisa, 2008

Quando aplicada a análise de regressão nas dimensões presteza mais, segurança mais, tangíveis mais, presteza menos, segurança menos e tangíveis menos, consideradas como variáveis preditoras (independentes) e na variável satisfação geral, considerada como variável dependente, foi encontrado um $\mathrm{R}^{2}=0,734$. Isto indica que $73,40 \%$ da variabilidade total da satisfação geral é explicada pelas variáveis independentes presteza mais, segurança mais, tangíveis mais, presteza menos, segurança menos e tangíveis menos.

\section{Figura 9: Análise de regressã(a) das dimensões de pesquisa para o modelo PRC}

\begin{tabular}{|c|c|c|c|c|c|c|}
\hline \multirow{2}{*}{\multicolumn{2}{|c|}{ Modelo }} & \multicolumn{2}{|c|}{$\begin{array}{l}\text { Coeficientes } \\
\text { Não-padronizados }\end{array}$} & \multirow{2}{*}{$\begin{array}{c}\text { Coeficientes } \\
\text { Padronizados } \\
\text { Beta }\end{array}$} & \multirow[b]{2}{*}{$\mathrm{t}$} & \multirow[b]{2}{*}{ Sig. } \\
\hline & & $B$ & Erro Padrãc & & & \\
\hline \multirow[t]{7}{*}{1} & (Constantes) & ,235 & ,102 & & 2,300 & ,025 \\
\hline & SegurMa & ,846 & ,240 & ,245 & 3,518 & ,001 \\
\hline & PrestezaMa & ,832 & ,292 & 197 & 2,853 & ,006 \\
\hline & Asp. Tang.Ma & ,132 & ,228 & ,049 &, 578 & ,566 \\
\hline & SegurMe &,- 629 &, 154 &,- 346 & $-4,087$ & ,000 \\
\hline & PrestezaMe & $-1,025$ & ,128 &,- 566 & $-7,999$ & ,000 \\
\hline & Asp. Tang.Me &,- 567 & ,164 &,- 260 & $-3,457$ & ,001 \\
\hline
\end{tabular}

* a) Variável dependente: Satisfação.

Fonte: dados da pesquisa, 2008

Foram realizados novos procedimentos estatísticos para adequação ao modelo PRC, ou seja, para verificar separadamente em qual grau cada uma das dimensões influencia a satisfação geral dos clientes das academias. A análise dos valores absolutos dos coeficientes de regressão estandardizados permitiu concluir que as variáveis Segurança Mais $(0,245)$ e Presteza Mais $(0,197)$; Presteza Menos $(-0,566)$ e Segurança Menos $(-0,446)$ apresentaram as maiores contribuições relativas para explicar a variável dependente satisfação geral. Quando observado os valores da estatística de $t$, as mesmas foram confirmadas.

Matzler et al. (2004) afirmaram que os atributos com coeficiente de penalidade significantemente superior ao coeficiente de recompensa são considerados obrigatórios, ou seja, se a satisfação com o atributo for baixa, a satisfação geral é influenciada, porém, se for alta, a satisfação geral não será influenciada. São considerados atrativos os atributos com coeficiente de recompensa com magnitude significantemente superior ao coeficiente de penalidade. Neste caso, quando a satisfação com o atributo for alta a satisfação geral será influenciada; mas isso não acontecerá quando a satisfação com o atributo for baixa. Quando o coeficiente de penalidade estiver próximo ao de recompensa, o atributo é unidimensional. Sendo assim, a satisfação geral será diretamente influenciada pelo nível de satisfação com o atributo. Os atributos considerados neutros são aqueles que não influenciam a satisfação geral independente do desempenho (Matzler et al., 2004; Picolo e Tontini, 2006).

A partir desta nova análise, foi possível identificar os atributos obrigatórios, unidimensionais, atrativos e neutros, baseando-se nos coeficientes de penalidade e recompensa com magnitudes mais significantes. 
Em relação às dimensões analisadas nas academias ALFA e BETA, baseando-se nas definições de Matzler et al. (2004) e Picolo e Tontini (2006), foi possível afirmar que os clientes consideraram como dimensões obrigatórias a presteza e os aspectos tangíveis, ou seja, a satisfação geral com as academias será influenciada somente quando o desempenho destas dimensões for baixo; além disso, avaliaram a dimensão segurança como unidimensional, isso significa que a satisfação geral é influenciada quando o desempenho for alto e quando for baixo (Ver Figura 10).

Figura 10: Análise do Contraste da Penalidade e da Recompensa

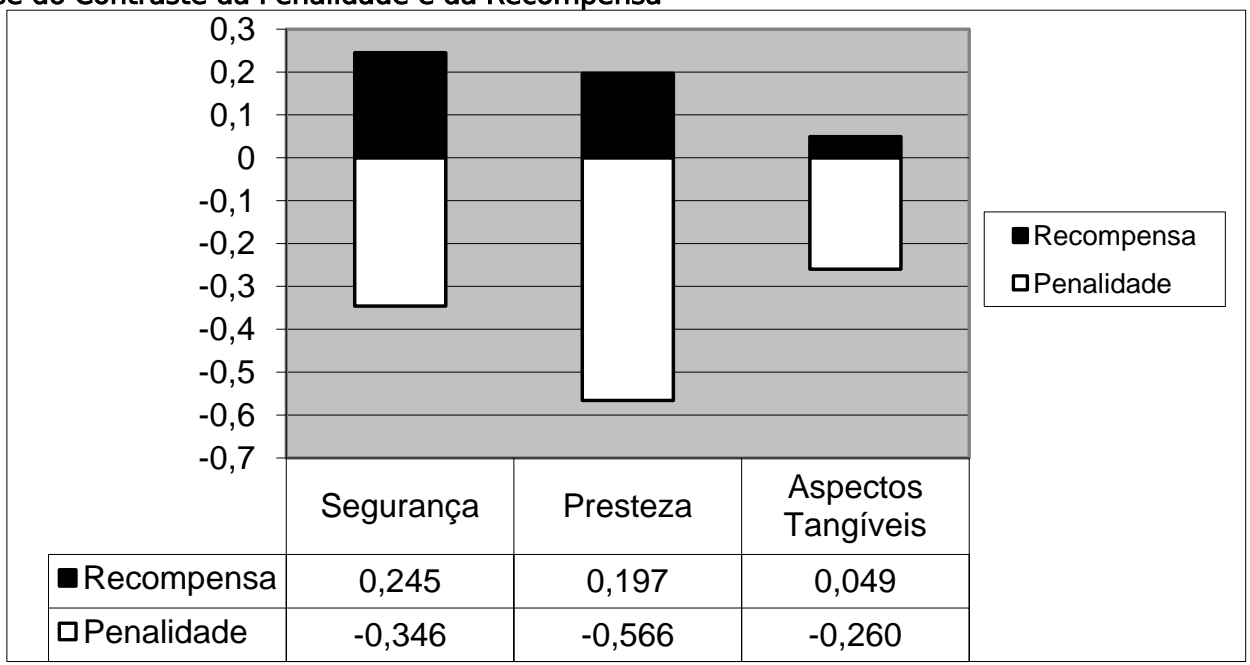

Fonte: dados da pesquisa, 2008

\subsection{Matriz de Importância vs Desempenho das Dimensões de Pesquisa}

O desempenho atual foi calculado pela diferença entre o desempenho da academia ALFA e o desempenho da academia BETA. O fato dos valores estarem todos abaixo de zero, significa que BETA possui atualmente melhor desempenho em todas as dimensões da qualidade (segurança, presteza e aspectos tangíveis). Os dados foram apresentados na Figura 11.

Figura 11: Importância estatística vs desempenho atual (ALFA - BETA)

\begin{tabular}{|l|c|c|}
\hline Dimensões & Importância Estatística & Desempenho Atual (ALFA - BETA) \\
\hline Segurança & 0,558 & $-0,52$ \\
Presteza & 0,587 & $-0,37$ \\
Asp. Tangíveis & 0,186 & $-0,03$ \\
\hline
\end{tabular}

Fonte: dados da pesquisa, 2008

Ao realizar uma matriz dos valores da importância estatística $(\beta)$ das dimensões, relacionados ao desempenho atual de cada uma delas, foi possível verificar de que forma cada academia (ALFA e BETA) deve agir para manter a satisfação geral em níveis altos. No eixo $x$ a linha divisória está em zero, ou seja, os valores acima de zero representam desempenho atual alto e os valores abaixo de zero, desempenho atual baixo. O eixo y representa a importância verificada estatisticamente e a linha divisória foi calculada a partir da média entre o valor de $\beta(0,4)$, conforme Figura 1 .

A Figura 12 mostra a matriz de importância vs desempenho para os atributos estudados neste trabalho. Foi possível perceber que no Quadrante I, no qual se encontram os atributos de alta importância e alto desempenho, não apareceram os atributos da academia ALFA. Isto significa que esta academia deve melhorar seus atributos para que possam os mesmos vir a encaixar-se nesta dimensão.

Os atributos presteza e segurança encontraram-se no Quadrante II, tendo alta importância, mas baixo desempenho, o que pode ser considerado um ponto fraco da academia ALFA. Deve haver um esforço para melhorar estes atributos, pois o aumento do grau de satisfação neles aumentará a satisfação geral com a academia. Estes atributos podem estar se tornando um diferencial para a academia BETA. 
Figura 12: Matriz de Importância vs desempenho para os Atributos das academias ALFA/BETA

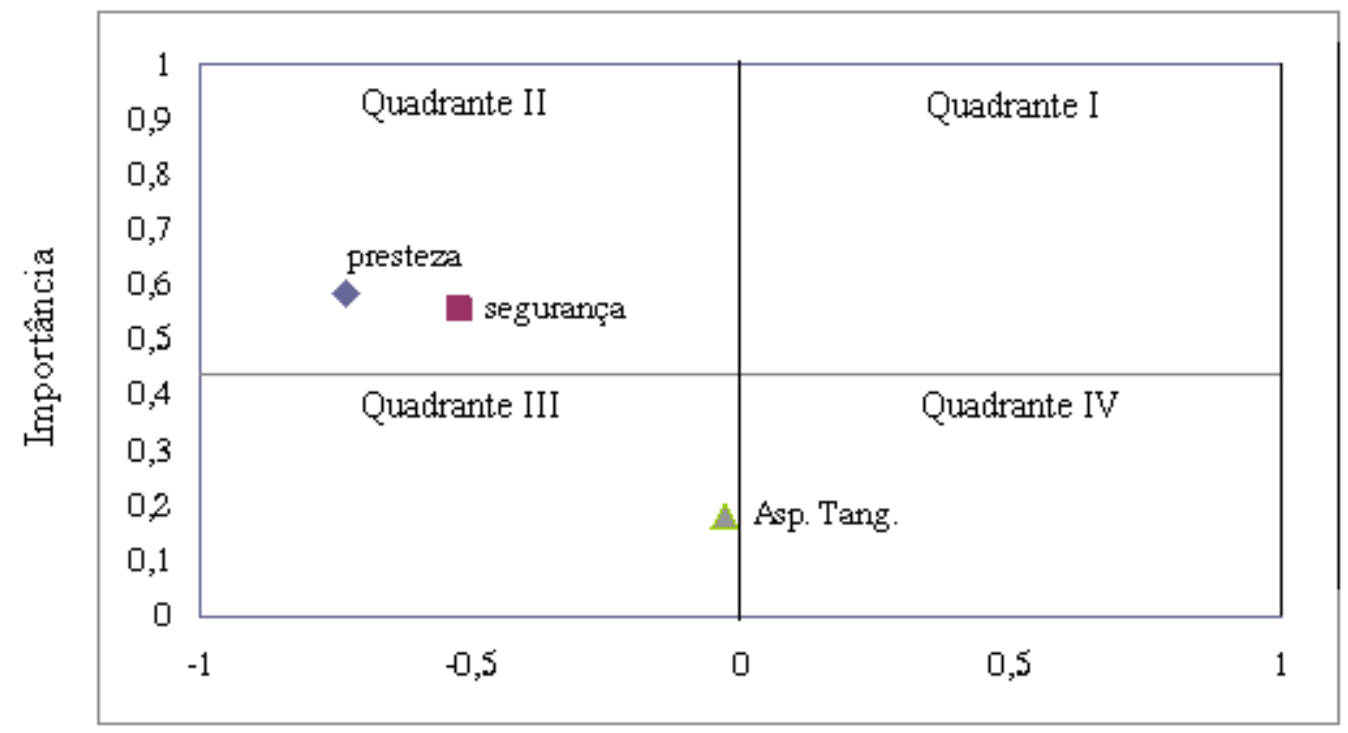

Desempenho

Fonte: dados da pesquisa, 2008

O Quadrante III conteve o atributo aspectos tangíveis. Por ter baixa importância e baixo desempenho, a academia ALFA não precisa concentrar esforços adicionais neste atributo. O Quadrante IV, assim como o Quadrante I, não apresentou enquadramento para os atributos da pesquisa.

Os resultados da matriz de importância vs desempenho foram confirmados pelas respostas à pergunta sobre a satisfação geral. A satisfação média com a academia ALFA foi de $M=5,657$ e com a academia BETA, $M=6,148$.

\section{Considerações finais}

O presente artigo utilizou-se do método de Análise do Contraste da Penalidade e da Recompensa (PRC) para determinar em que intensidade cada uma das dimensões da qualidade influencia a satisfação geral dos clientes nos serviços prestados em duas academias de ginástica. Para isso, foi utilizado o modelo SERVQUAL de 22 questões, sugerido por Parasuraman et al (1988), levando-se em consideração as três dimensões da qualidade que explicam as variações na satisfação geral dos cientes: segurança, presteza e aspectos tangíveis.

Além disso, com a aplicação do PRC, foi possível observar a combinação entre a recompensa e a penalidade de cada dimensão, para indicar de que forma elas se relacionam com a satisfação geral. A presteza e os aspectos tangíveis foram considerados atributos obrigatórios, ou seja, a satisfação geral será influenciada quando o desempenho nestes atributos for baixo, mas o mesmo não ocorrerá quando o desempenho for alto. A segurança foi considerada como atributo unidimensional, pois influencia diretamente a satisfação geral, aumentando-a quando o desempenho á alto e reduzindo-a, quando é baixo.

A partir da análise da matriz de importância vs desempenho, foi possível traçar um comparativo entre a performance das academias analisadas. A academia BETA possui melhor desempenho em todas as dimensões. Para que a academia ALFA se torne mais competitividade e aumente a satisfação geral de seus clientes, ela deve direcionar esforços para melhorar nas dimensões segurança e presteza, por possuírem grande importância, porém baixo desempenho se comparadas à BETA.

Como o presente estudo consiste de uma pesquisa exploratória e descritiva de dois casos, é possível indicar como limitação a amostra reduzida. O questionário foi aplicado em 63 clientes, porém, segundo orientações de Hair Jr. et al. (2009), são necessários cinco ou mais observações para cada variável a ser analisada. Sendo assim, a quantidade recomendada seria de 75 clientes.

Na seleção da amostra é possível que tenha havido outra limitação, já que a escolha aconteceu levando em consideração a freqüência em que o serviço das academias é utilizado e não há quanto tempo o 
respondente é cliente. Fica a sugestão para um próximo estudo, que verifique se este atributo influencia os resultados tanto no que tange as dimensões da qualidade, como na satisfação geral dos clientes.

\section{Referências}

Anderson, E. W., Mittal, V. (2000). Strengthening the satisfaction-profit chain. Journal of Service Research, 3 (2), pp. 107-120, Nov. doi: 10.1177/109467050032001

Berry, L. (1996). Serviços de satisfação máxima: guia prático de ação. Rio de Janeiro: Campus.

Bateson, J. E. G. e Hoffman, K. D. (2001). Marketing de serviços (4a ed.). Porto Alegre: Bookman.

Bolton, R. D., Drew, J. H. (1991). A multistage model of customers' assessment of service quality and value. Journal of Consumer Research, 17 (4), pp. 375-84. doi: 10.1086/208564

Brandt, R. D. (1987). A procedure for identifying value-enhancing service components using customer satisfaction survey data. In: Surprenant, C. (Ed.). Add Value to Your Service. Chicago: American Marketing Association.

Fitzsimmons, J. A. e Fitzsimmons, M. J. (2000). Administração de serviços: operações, estratégia e tecnologia de informação (2a ed). Porto Alegre: Bookman.

Garver, M. S. (2003). Best practices in identifying customer-driven improvement opportunities. Industrial Marketing Management, 32, pp.455-466. doi: 10.1016/S0019-8501(02)00238-9

Grönroos, C. (1984). A service quality model and its marketing implications. European Journal of Marketing, 18 (4), pp. 36-44. doi: 10.1108/EUM0000000004784

Hair, J. F. et al. (2005). Análise multivariada de dados (5a. ed.). Porto Alegre: Bookman.

Hair JR, J. F. et al. (2009) Análise multivariada de dados (6a ed.). Porto Alegre: Bookman.

Kano, N. et al. (1984). Attractive quality and must-be quality. The Journal of the Japanese Society for Quality Control, Abr., pp. 39-48.

Maroco, J. (2003). Análise estatística: com utilização do SPSS. Machester: Edições Silabo.

Martilla, J. A. \& James, J. C. (1977). Importance performance analyses. Journal of Marketing. 9, p. 41-77.

Matzler, K., Bailon, F., Hinterhuber, H. H., Renzl, B. \& Pichler, J. (2004). The asymmetric relationship between attribute-level performance and overall customer satisfaction: a reconsideration of the importance-performance analysis. Industrial Marketing Management, 33 (4), pp.271-277. doi: 10.1016/S0019-8501(03)00055-5

Mittal, V., Ross Jr, W. T. \& Baldassere, P. M. (1998). The asymmetric impact of negative and positive attribute-level performance on severall satisfaction and purchase intentions. Journal of Marketing. 62, pp. 33-47, jan. doi: 10.2307/1251801

Parasuraman, A., Zeithaml V. A. \& Berry, L. L. (1985). A conceptual model of services quality and its implication for future research. Journal of Marketing, 49 (4), pp. 41-50. doi: 10.2307/1251430

Parasuraman, A., ZeithamI V. A. \& Berry L. L. (1988). SERVQUAL: A multiple-item scale for measuring consumer perceptions of service quality, Journal of Retailing, 64 (1), pp. 12-40.

Picolo, J. D. e Tontini, G. (2006). Análise do contraste da penalidade e da recompensa (PRC): identificando oportunidades de melhoria em um serviço. In: Anais do Encontro da Associação Nacional dos Programas de Pós-Graduação em Administração, Salvador, BA, Brasil, 30.

Ting S. C.; Chen, C. N. (2002). The asymmetrical and non-linear effects of store quality attributes on customer satisfaction. Total Quality Management, 13 (14), pp. 547-569.

Tontini, G. (1996). Administração estratégica da qualidade: a chave para a qualidade e a produtividade. Revista de Negócios, 1 (3), pp. 5-12.

Tontini, G. (2003a). Como identificar atributos atrativos e obrigatórios para o consumidor. Revista de Negócios, 8 (1), pp. 19-28. 
Tontini, G. (2003b). Determining the degree of satisfaction of customer requirements: a modified Kano method. California Journal of Operations Management, 1 (1), pp. 95-103.

Tontini, G. (2003c). Deployment of Customer Needs in the QFD Using a Modified Kano Method. Journa/ of Academy of Business and Economics, 2 (1), pp. 103-115.

Tontini, G. e Silveira, A. (2005). Identification of critical attributes of success in products and services: an alternative to importance - performance analysis. In: Proceedings of BALAS Annual Conference, Madrid, ES. 